\title{
TEJIENDO LA RED: CIRCUITOS COMERCIALES, INMIGRANTES SIRIO- LIBANESES Y EMPRESARIOS NACIONALES EN EL CARIBE COLOMBIANO Y EL ATRATO (1880-1930)
}

\section{Ana Milena Rhenals Doria}

\begin{abstract}
Resumen
Este articulo reconstruye el circuito comercial existente entre Cartagena, las provincias del Sinú y el Atrato, destacando las particularidades de estos espacios, los empresarios nacionales y locales que actuaban en ese escenario antes de la llegada de los inmigrantes sirio-libaneses, y la manera como se fueron haciendo presente estos últimos en este contexto socio-económico.
\end{abstract}

Palabras Claves: circuito comercial, sirio-libaneses, Caribe colombiano, Atrato.

\begin{abstract}
This paper reconstructs the commercial route between Cartagena, the provinces of Sinú and the Atrato. It highlights the peculiarities of these areas, the national and local businessmen who worked on these contexts before the arrival of the SyrianLebanese immigrants, and how they emerged and took advantage of the socioeconomic conditions of the previously mentioned commercial route.
\end{abstract}

Keywords: marketing network, Syrian-Lebanese, Colombian Caribbean, Atrato.

\section{Introducción}

La presencia de inmigrantes sirio-libaneses en la realidad colombiana ha sido uno de los fenómenos sociales más significativos en términos del desarrollo comercial y económico de las distintas regiones donde se asentaron. Tal vez el espacio geográfico donde es más notable su impacto es en el circuito comercial existente entre las provincias del Sinu (Loríca, Cereté), el Atrato (Quibdó) y el puerto de Cartagena. Aunque diversos autores han llamado la atención sobre esta importante presencia, al intentar explicar el exitoso proceso de posicionamiento económico de estos actores sociales desdibujan las realidades contextuales en las cuales éstos se hallaban inmersos. De manera mecánica caracterizan los

\footnotetext{
* Artículo de investigación tipo 2: de reflexión según Colciencias. Hace parte de la tesis doctoral que la autora viene adelantando sobre inmigración sirio-libanesa en el Caribe colombiano y el Atrato.

** Historiadora de la Universidad de Cartagena, y candidata a doctora en Historia de América Latina de la Universidad Pablo de Olavide de Sevilla - España. Es miembro del grupo de investigación Sociedad Cultura y Política en el Caribe colombiano del Instituto Internacional de Estudios del Caribe. E-mail: mirhedo@yahoo.es.
} 
espacios como lugares en los que el desarrollo de actividades comerciales era casi nulo antes de la llegada de los mismos; señalando que es solo después de la llegada de los sirio-libaneses y su injerencia en la actividad comercial que las poblaciones logran un despegue económico y un fluir comercial. Un claro ejemplo de esta tendencia se encuentra en el texto "Lorica, una colonia árabe a orillas del Río Sinú", escrito por el economista Joaquín Viloria. Suscribiendo las apreciaciones del periodista Antolín Díaz y las del viajero B. Le Roy Gordon, Viloria, al caracterizar la vida económica de Lorica, termina hablando de una incipiente vida comercial que solo será dinamizada con la llegada de inmigrantes sirio-libaneses. (Viloria, 2003. p. 35,50).

En igual sentido se expresó José Francisco García Calume al describir el contexto económico de Cereté. Según este descendiente sirio en esta localidad, antes de la llegada de los mencionados inmigrantes, "solo se cultivaba para el pancoger, sin criterios comerciales." (García Calume, 1990, p.4). Y más recientemente la escritora Soad Louis Laka, en el texto "Los inmigrantes árabes en los valles del Sinú", reproduce esta misma perspectiva. Al intentar describir el proceso de establecimiento de un grupo de árabes en la población de Ciénaga de Oro, categóricamente afirma:

"Ciénaga de Oro, un pueblo donde no pasaba nada, casi todos los días eran lo mismo, uno detrás del otro todos los días el sol golpeando las calles de arena. Cualquiera se podía sentar en la puerta de su casa y quedarse allí de seis a seis que no pasa nada". (...) "Todo lo que estoy contando sucedió antes de la llegada de los árabes, luego ellos llevaron los almacenes, las historias de amores, los encajes, las telas, los botones (...)". (Louis, 2008, p. 12)

Contrario a la imagen que se ha querido cimentar de estos espacios con una incipiente vida económica, marcada por la inexistencia de actividades comerciales antes de la llegada de los inmigrantes sirio-libaneses, lo que parece caracterizar a estos contextos durante toda la segunda mitad del siglo XIX es una intensa actividad comercial liderada por comerciantes nacionales que en estrecha relación con las dinámicas que estaba experimentando Cartagena y el Atrato en términos comerciales fueron definitivas para el desarrollo de circuitos mercantiles locales, regionales e internacionales. De modo que el camino recorrido por los inmigrantes sirio-libaneses para su establecimiento definitivo remite a un mundo de posibilidades, beneficios, vínculos e interacciones sociales que necesitan ser reconstruidas a partir de cuestionamientos sobre el accionar de estos inmigrantes desde su llegada hasta su total inserción en la vida económica y social. ¿Por qué Cartagena, la Provincia del Sinú y el Atrato?, ¿quiénes y cuántos?, son algunas de las preguntas que este articulo intenta resolver. El propósito central del mismo es reconstruir el circuito comercial existente entre Cartagena, las provincias del Sinú y el Atrato, destacando las particularidades de estos espacios, los empresarios 
nacionales y locales que actuaban en ese escenario antes de la llegada de los inmigrantes, y la manera como se fueron haciendo presente estos últimos en este contexto socio-económico.

\section{El Contexto}

A finales del siglo XIX Colombia logró integrarse de manera estable a la economía internacional como producto del ascenso de las exportaciones de café, lo cual significó la consolidación del sector exportador, el mejoramiento en los transportes y una mayor integración al mercado interno, originándose una época de apogeo económico. Esta nueva etapa de la economía acrecentó la actividad comercial en los principales puertos marítimos del Caribe, puertas de salida de los productos enviados del interior del país y entrada de gran cantidad de mercancías del exterior. (Bejarano, 1997, pp. 231-235). El permanente y continuo movimiento de productos de exportación y la comercialización de diferentes mercancías, reactivaron la agricultura y el comercio en la región, lo cual generó unas dinámicas comerciales que ofrecían mayores oportunidades de negocios en la zona. (Ver mapa No. 1).

Desde esta época, la región Caribe estuvo enmarcada en unas condiciones económicas en vía de mejoramiento que resultaron favorables para el establecimiento de estos inmigrantes; así como también la zona del Atrato que, además de su estrecha vinculación con la vida comercial del puerto de Cartagena que la hacía ser comercialmente parte de la región Caribe, estaba viviendo todo un auge en la industria del platino y la agricultura, hecho que la convirtió en un punto atractivo para inversionistas y comerciantes. A los factores ya descritos se le suma la fertilidad de sus suelos que consolidaba el cultivo de productos como el banano, caucho y el tabaco, e incidieron en la estimulación de las relaciones comerciales del país con el mercado internacional. Además, como las mejoras en el transporte fluvial fueron más tempranas que las terrestres, las tierras bajas cercanas a los ríos navegables se articularon más rápido al mundo exterior entre 1860 y 1910, cuando la economía exportadora tomo fuerza en el país a través de la exportación de café y la Costa se convirtió en centro de acopio de productos agrícolas, generándose una actividad portuaria que identificaría las principales ciudades del Caribe como Cartagena y Barranquilla. (Zambrano, 1998, p. 41).

La Provincia del Atrato, que mantenía desde tiempos coloniales una relación con el puerto Cartagena, en igual sentido presentó unas características y dinámicas económicas que se fortalecieron desde la segunda mitad del siglo XIX. Su condición geográfica privilegiada, que la mantenía bañada por una importante vía fluvial que se conecta con el Mar Caribe, sirvió de camino para que la riqueza forestal y aurífera de sus suelos se desarrollara con mayor intensidad. La explotación de estos recursos naturales trajo un fluir comercial que generó un 
mercado interno con epicentro en Quibdó que tiene un auge en la década del setenta del siglo XIX1'. (González, 2003, pp. 62 -68).

\section{Mapa No. 1 \\ Producción de la región y productos que se movían por el circuito comercial}

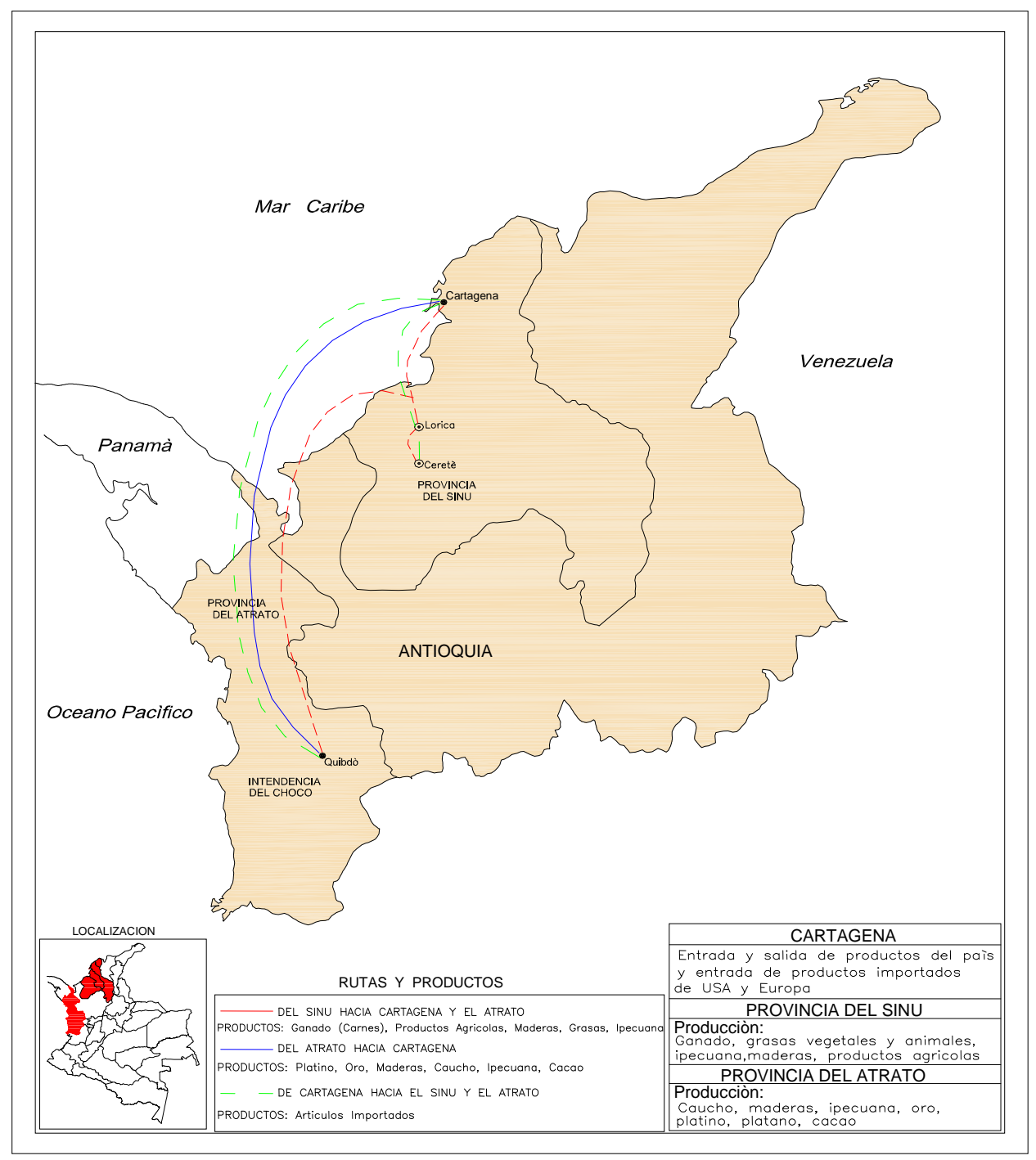

A finales del XIX la bonanza minera despertó expectativas con el platino, se iniciaría lo que se ha llamado "la fiebre del oro", así como también el fortalecimiento de la producción del caucho, la tagua, ipecuana y maderas finas, que convirtieron al Atrato en una gran zona de explotación de materias primas

\footnotetext{
${ }^{1}$ En Quibdó la primera etapa de la navegación a vapor se desarrolla desde 1850-1875, y se regulariza con apoyo estatal entre 1875-1910, adelantando el comercio entre Quibdó y Cartagena. Archivo Histórico de Cartagena, (en adelante A.H.C), Emigdio Palau, "Informe al Congreso de la República", Diario de Bolívar, $\mathrm{N}^{\circ} 1130$, Cartagena, 13 de mayo de 1875, p. 425.
} 
exportables a los mercados de Europa y los Estado Unidos de América. Estas realidades intervinieron en el auge comercial que vivió el Atrato en las décadas del setenta y ochenta, acentuando su relación con el mercado internacional. Durante el siglo XX esta zona sigue mostrando unas condiciones especiales como lo fueron, primero, la declaratoria en el año de 1907 de libre exportación de platino y, segundo, el convertirse en la principal fuente mundial de platino, que marcó un periodo de prosperidad sin referentes. Se pasó de vender a 50 dólares la onza troy ${ }^{2}$ de platino a 115 dólares. (González, 2003, pp. 77-79).

Cartagena, Lorica y Cereté, que para comienzos del siglo XX pertenecían a la división político-administrativa del Departamento de Bolívar, se encuentran ubicadas en la región Caribe en la zona norte de Colombia. Cartagena, una de las ciudades portuarias más importantes del país, se halla situada en el noroeste de la región sobre el mar Caribe, condición geográfica que le ha dado preponderancia a nivel portuario y comercial desde tiempos coloniales. Las poblaciones de Lorica y Cereté, por su parte, están ubicadas al noreste en el actual departamento de Córdoba a sesenta kilómetros de distancia la una de la otra, y ambas bañadas por el río Sinú. Lorica, además de estar atravesada de norte a sur por el río, está a 40 kilómetros del litoral Caribe. El río Sinú, que atraviesa las dos poblaciones y tiene su desembocadura en la bahía de Cispatá hacia el mar Caribe, permitió el desarrollo una ruta marítimo-fluvial y conectó geográfica y comercialmente a Cartagena y la provincia del Sinú. Quibdó, población situada en la región Pacifica colombiana, en la antigua Provincia del Atrato, hoy Departamento del Chocó, está ubicada a la margen derecha del rio Atrato, cuya desembocadura esta en el Golfo de Urabá en el Mar Caribe a una distancia de 342 kilómetros de la desembocadura ${ }^{3}$. Esta característica significó también la unión comercial con el puerto de Cartagena, conformando y articulando así un triangulo comercial entre las poblaciones de Cartagena, Quibdó (Atrato), Lorica y Cereté (Sinú), que fue fundamental tanto para el proceso de recuperación económica de la primera, como para la consolidación de las actividades comerciales de los tres restantes distritos.

Lorica y Cereté eran los centros de acopio, comercialización y salida hacia Cartagena y el Atrato de toda la gama de productos agrícolas que provenían del resto de poblaciones que integraban la provincia del Sinú, siendo las más importantes Momil, Chimá, Chinú, Ciénaga de Oro, Sahagún, entre otras. Estas localidades se vinculaban al circuito comercial a través de la producción de ganado, plátanos, raicillas, ipecuana, manteca. Quibdó, por su parte, era el epicentro de un mercado interno que suministraba productos agrícolas y mineros

\footnotetext{
${ }^{2}$ Este tipo de onza sólo es usada en joyería, orfebrería y numismática para pesar metales preciosos. Es la doceava parte de una libra troy y equivale a 31,1034768 gramos.

${ }^{3}$ Biblioteca Nacional de Colombia. (en adelante B.N.C), "El Chocó”, El Chocoano, periódico miscelánico, Quibdó, 10 febrero 1899, N 4, p. 1. El Atrato nace en la cordillera occidental de los Andes y desemboca en el mar Caribe, recorre gran parte del departamento de Chocó.
} 
que mantenían la actividad comercial de poblaciones como Condoto, Istmina, Budó, contactándose con el importante puerto del Caribe ${ }^{4}$.

Esta realidad económica intensificó el dinamismo productivo existente entre la ciudad y las provincias, y aumentó el transporte fluvial por los ríos Sinú y Atrato. Aunque el movimiento comercial y de extracción de productos de ambas provincias hacia Cartagena venía desarrollándose desde tiempos coloniales, el despliegue del transporte y comercio fluvial empezó a crecer notablemente con el establecimiento de casas comerciales, empresas navieras, financieras y comisionistas de capital nacional desde mediados del siglo XIX. Durante este siglo la relación existente entre Cartagena y las provincias del Atrato y Sinú cobra mucha más fuerza e importancia con motivo de la prolongada crisis económica que vivió Cartagena. Como ha sido ampliamente referenciado por la historiografía, Cartagena desde inicios del siglo XIX había perdido el poderío económico que desde los tiempos de la colonia la habían caracterizado como uno de los puertos más importante del Caribe hispánico; la navegabilidad del Canal del Dique se había perdido y eso representó la incomunicación del puerto con la arteria de comunicación más importante del país, como lo era el río Magdalena. Fue, entonces, la navegabilidad por los ríos Sinú y Atrato la que le dio un aire y movimiento comercial durante y después de la crisis ${ }^{5}$.

El amplio movimiento comercial y la posibilidad de desarrollo de riquezas de poblaciones como Lorica, Quibdó o Cereté serian fundamentales para su recuperación. En otras palabras, mientras Cartagena se hallaba sumida en su crisis, el mundo económico de las provincias del Sinú y el Atrato mantenían un interesante dinamismo. La provincia del Sinú, con Lorica y Cereté como puertos fluviales y comerciales de gran importancia, y en el Atrato Quibdó como principal puerto fluvial y centro agrícola y minero, desde la segunda mitad el siglo XIX registraron un auge económico ${ }^{6}$. Estas provincias, referenciadas desde los tiempos de la conquista española como unas zonas de abundante riqueza

\footnotetext{
${ }^{4}$ Es importante anotar que las diferentes poblaciones de la provincia del Sinú tenían una producción agrícola que se movía y comercializaba por toda la región y sobre todo en Cartagena, así mismo las zonas de Tolú, Corozal, Sincelejo, pertenecientes a las sabanas de Bolívar integraban este circuito comercial. Para la segunda mitad del siglo XX las relaciones comerciales tanto del Sinú como del Atrato no estaban vinculadas exclusivamente con el Caribe, sino que se fortalece la relación económica con el departamento de Antioquia a través de una red de caminos, que ayudan a superar las dificultades de sedimentación de los ríos. Ver: B.N.C, Chocó: Geografía económica de Colombia, Tomo VI, Chocó, Contraloría General de la Republica, Bogotá, 1943. DIAZ D, Fernando, Cultura del Bajo Sinú: Tradición, educación y cambio, Alcaldía Municipal de Lorica, Santa Cruz de Lorica, 2004.

${ }^{5}$ En el año de 1871, se estableció que "por estos ríos pasaban de 30.000 a 40.000 toneladas de mercancías de importación y exportación, fuera a lo que pertenece al comercio interior propiamente dicho, que no es de poca consideración sobre todo en Bolívar. Este Estado produce ya más de 100 mil quintales de tabaco, mas de 40.000 de algodón y exporta caucho, ipecuana, bálsamo, maderas de tinte y construcción, y otros artículos". B.N.C. "Estados unidos de Colombia, lei (sic) sobre fomento de tierras baldías", Bogotá, Imprenta Medardo Rivas, 1871, p. 6.

${ }^{6}$ Desde la prensa se logra ver como la relación Cartagena-Quibdó-Sinú fue una realidad desde tiempos coloniales, varios artículos resaltan este vínculo comercial. B.N.C, "Cartagena y el Chocó", $A B C$, Quibdó, 25 julio 1927, págs. 1-4. "El Sinú y el Atrato", ABC, Quibdó, 2 enero 1930. A.H.C, "Movimiento comercial", Diario de la Costa, 12 enero 1916.
} 
aurífera, resultaron ser lugares llenos de un gran potencial de riquezas naturales prolijo y apto para su explotación. Esta característica les permitió seguir manteniendo durante el siglo XIX una relación comercial con el puerto de Cartagena como proveedoras de alimentos, productos agrícolas y forestales para abastecer a la ciudad y para su exportación.

La actividad comercial que se mantenía entre estos tres puntos, además de generar el intercambio de productos, produjo un movimiento de gentes que de alguna manera contribuyó a que las ideas sobre la riqueza mineral que existía en el Sinú y el Atrato continuaran y se fortalecieran para los primeros años de la segunda mitad del siglo XIX, cuando el interés económico por estas provincias se potenció debido a las explotaciones de maderas, recursos agrícolas y auríferos de platino (Atrato) ${ }^{7}$. Como resultado del fortalecimiento de estas imágenes, todas estas provincias se convirtieron en puntos de atracción para los comerciantes de la región y el país.

\section{El rol de los empresarios nacionales}

Tanto en el Caribe como en el Atrato, actividades como el comercio, la exportación de productos agrícolas, y la creación de compañías de transporte fluvial habían estado en manos de un grupo de personas y familias de origen nacional desde el siglo XIX. Estos iniciaron a pequeña escala una actividad comercial que los conectaba tanto con el exterior como con las diferentes provincias del Caribe colombiano. Un ejemplo de esto es la elite empresarial de Barranquilla que desde 1875 estaba vinculada a los más variados negocios, la navegación a vapor, las obras de comunicación de Barranquilla con el mar y las empresas transportadora. (Solano \& Conde, 1993, p. 16). Así mismo, desde el año 1800 , los empresarios de la ciudad portuaria de Santa Marta fueron pioneros o continuadores de varios proyectos de trascendencia nacional, y algunas de sus empresas regularizaron la navegación a vapor por el Río Magdalena, invirtiendo en negocios de navegación fluvial y marítima; además de dedicarse a la importación y exportación de productos agrícolas (Viloria 2001, p. 14).

A los empresarios barranquilleros y samarios se sumaron los cartageneros. El movimiento de mercancías y productos agrícolas y auríferos, fue una constante entre el puerto de Cartagena y las zonas del Sinú y el Atrato, desde tiempos coloniales, por tanto no es extraño que desde el siglo XIX la economía cartagenera estuviera decididamente fundamentada en la explotación de los recursos naturales de las regiones próximas al Sinú y el Atrato. Entre 1850 y 1887, el caucho, la tagua, la ipecuana, el platino y las maderas habían permitido que los comerciantes establecidos en Cartagena se capitalizaran y posteriormente establecieran sucursales de casas comerciales en localidades como Lorica, Cereté y Quibdó, y viceversa (González, 1997, pp. 73-74).

\footnotetext{
${ }^{7}$ En el año de 1873, el agente comercial norteamericano F.W.P Smith, señalaba que hasta 4000 hombres estaban entregados a la explotación de caucho en los bosques del Sinú y el Chocó.
} 
Empresarios como Rafael del Castillo, José Vicente Mogollón y las familias Martínez, Pombo, Bossio y Zubiria, desde finales del siglo XIX mantenían redes comerciales entre la ciudad, el interior de la región, el centro del país, Norte América y Europa. En su gran mayoría tenían como soporte las empresas navieras que les daban la posibilidad de monopolizar la compra de productos naturales de la región del Atrato Urabá y Sinú para surtir a Cartagena de víveres. En las zonas del Atrato y el Sinú comerciantes e inversionistas como los Perea, Posso, Ferrer, Asprilla, Mosquera, Martínez, Burgos, Rodríguez, junto a los cartageneros, mantenían una activa vida comercial representada en la importación y exportación de artículos y productos agrícolas y mineros, así como también la navegación por los ríos Sinú y Atrato hasta el puerto de Cartagena (González, 2003, pp.82-84).

Todas estas actividades económicas de los empresarios del Caribe colombiano $y$ el Atrato demuestran que fueron capaces de crear o formar unas redes comerciales adecuadas para el desarrollo económico de la zona, en busca de superar la crisis comercial en que los sumía la continua y sistemática obstrucción del Canal del Dique, que mantenía a la ciudad comercialmente incomunicada con el interior del país. En la década de 1880 en Cartagena se emprende todo un proyecto nacional de recuperación representado en los trabajos de dragado y canalización del Dique, la construcción del muelle marítimo de la Machina y de la línea férrea que uniría a la ciudad con el puerto fluvial de Calamar. Estas obras facilitaron la conexión de la ciudad a costos razonables con el río Magdalena, ayudándola a mejorar su actividad económica.

Estas circunstancias la constituyeron, por ejemplo, en un punto clave para el desarrollo económico nacional. La región Caribe, en comparación con el resto del país, se convirtió durante las últimas décadas del siglo XIX en un eje de desarrollo económico que se destacó por su producción agrícola, la ganadería, la minería, explotación de bosques y el transporte fluvial y marítimo que permitieron unas vías de comercio que lograban conectarla con el interior del país y facilitaban una relación más estrecha con el exterior. (Posada, 1998, p.439). Al finalizar el siglo XIX, Cartagena mostraba ya unos signos notables de recuperación económica ${ }^{8}$, expresados en una activa vida comercial y empresarial liderada por comerciantes y empresarios locales que se movían entre las importaciones, exportaciones, el comercio al detal, las comisiones, el transporte fluvial y las inversiones en la agricultura, la ganadería y la minería. Según los historiadores Jorge Restrepo y Manuel Rodríguez Becerra, esta ciudad desde los tiempos coloniales se

\footnotetext{
${ }^{8}$ La actividad comercial del puerto de Cartagena mostró un marcado ascenso durante los primeros años del siglo XX. En un artículo publicado en el periódico La Verdad se realiza un balance del movimiento de entradas y salidas de productos por el puerto durante los años de 1909 a 1913, en los que se hace notorio el aumento de esta actividad. En el año de 1909 se registró 30.319 bultos; en 1910, 93.221; 1911, 94.315; 1912, 158.065; hasta llegar a la suma de 253.536 bultos en el año de 1913. la mayoría de los productos que se movían eran café, caucho, ipecuana, raicilla, tagua, tabaco, plátanos, frutas, cueros, carne, madera y mantecas vegetal y animal; la gran mayoría extraídos de las poblaciones próximas al Sinú y el Atrato. A.H.C. "Cartagena", La Verdad, Cartagena, mayo 14 de 1913. "Resumen de las exportaciones", La Época, Cartagena, enero 13 de 1913.
} 
caracterizó por poseer una fuerte comunidad de comerciantes, que incluso durante el siglo XIX, en medio de la crisis que vivía la ciudad, siguieron dominando la vida económica, y muchos de ellos exploraron fuentes de riqueza en el río Atrato ${ }^{9}$ y, por supuesto, en el río Sinú (Restrepo y Rodríguez, 1986 p. 104).

Entre los comerciantes más destacados en Cartagena, para finales del siglo XIX y comienzos del XX, se pueden señalar a los hermanos José Joaquín y Esteban de Pombo, Carlos Vélez Daníes, Rafael Del Castillo, entre otros. Desde el año de 1883, los hermanos Pombo fundaron la casa "Pombo Hermanos", dedicada a la exportación e importación de productos agrícolas (arroz, mantecas de cacao y cerdo), la venta de mercancías de todo tipo, y eran agentes de casas comerciales establecidas en Nueva York. El empresario Carlos Vélez Daníes, propietario de la casa "Vélez Danies \& Compañía", realizaba compras y ventas de productos agrícolas (grasa animal y vegetal), materiales de construcción, y se dedicaba a la realización de préstamos. Sin embrago, su actividad económica más destacada la constituyó la cría de ganado en la región del Sinú. De esta zona sacaba un importante número de cabezas de ganado en pie que eran exportados, a través del puerto de Cartagena, hacia México, Cuba, Panamá, Perú, Santo Domingo y Venezuela. Esta experiencia le permitió convertirse posteriormente en uno de los principales socios del proyecto del Packing House de Coveñas ${ }^{10}$ a comienzos del siglo XX (Restrepo y Rodríguez, 1986, pp. 89-99).

Rafael del Castillo, reconocido empresario de Cartagena, a través de su firma "Rafael del Castillo \& Co", creada a mediados del siglo XIX (1861), se dedicó a actividades económicas como el comercio, la ganadería y el transporte fluvial. Del Castillo, quien tenía su casa matriz establecida en Cartagena y sucursales en Estados Unidos, exportaba e importaba una amplia gama de productos que eran redistribuidos hacia el interior del país, las sabanas de Bolívar y el Sinú, al tiempo que realizaba operaciones relacionadas con préstamos y representación de casas comerciales internacionales de Europa y Norte América. (Ripoll, 2000, pp.7-10). Desde el año de 1870, Nicolás de Zubiría, empresario establecido en Cartagena, puso en funcionamiento el vapor "Bolívar" de 120 toneladas de capacidad, que navegaba entre Cartagena y las poblaciones del Sinú y el Atrato dos veces al año, mientras Bartolomé Martínez Bossio mantuvo una amplia gama de negocios con la zona del Atrato, siendo el transporte fluvial una de sus actividades empresariales más consolidadas (Díaz, 1994, p. 42).

En las poblaciones de Lorica y Cereté también hubo presencia de un gran grupo de comerciantes nacionales que realizaron un conjunto de actividades económicas que serian vitales para la posterior inserción de los inmigrantes siriolibaneses al circuito comercial de esta región. Estas poblaciones fueron de las más

\footnotetext{
${ }^{9}$ Este río constituía la vía, columna vertebral, de la actividad comercial entre el Chocó y Cartagena.

${ }^{10}$ Este fue un proyecto de producción ganadera, desarrollado en los primeros años del siglo XX, para el procesamiento y exportación de carnes en la zona del Sinú, cuyo fin era ampliar los mercados de exportación con la instalación de un frigorífico en la población de Coveñas.
} 
dinámicas comercialmente hablando durante el siglo XIX en la Provincia del Sinú. Su actividad comercial estuvo encabezada inicialmente por empresarios y negociantes locales y nacionales dedicados a la ganadería, la agricultura y el comercio Favorecidos por la presencia del río, mantenían contacto con el puerto de Cartagena y el exterior, lo cual se registró con mayor fuerza desde el siglo XIX. Cereteanos como José de la Cruz Puche Brango y el empresario Manuel Burgos, se destacaron como agentes promotores de la actividad económica de la población. José de la Cruz, reconocido como uno de los iniciadores en la población de la importación de mercancías de Europa y los Estados Unidos y de la exportación al viejo continente de raicillas de ipecuana, fue uno de los pioneros de la navegación fluvial entre Cartagena y Cereté. Este empresario contaba con barquetonas particulares (San José y Santa Paula) que embarcaban pasajeros y cargas para Cartagena, Quibdó, Tolú, Montería, Lorica y Ciénaga de Oro. (Rodríguez, 2001, p.4). Para la misma época el empresario cereteano Manuel Burgos fundó la Hacienda Berástegui, que fue una de las empresas agrícolas más importantes de la región, y se dedicaba a la producción de azúcar y la exportación de tabaco, caucho y ganado. (Nacimento, 1916-1919, p. 10). Otros comerciantes cereteanos como José A. Rodríguez, Francisco Padrón, entre otros, mantenían relaciones con importantes casas de comercio de Cartagena como "Rafael del Castillo \& Co", a través de las cuales recibían mercancías importadas provenientes de Europa y Norteamérica ${ }^{11}$.

La dinámica comercial de Cereté se dio con mayor fuerza en los años finales del siglo XIX y principio del XX, al constituirse en centro de mercadeo y acopio subregional, y funcionar como puerto de embarque de los productos agrícolas con destino al puerto de Lorica, como primera escala y luego a Cartagena. Esta circunstancia le fue otorgando un perfil gradualmente ascendente en cuanto actividades comerciales se refería, que si bien no tenían la misma dimensión y movimiento registrado en Lorica, le significó a Cereté la posibilidad de tener figuración en la vida comercial de la región.

Toda esta actividad empresarial se vio reflejada en la proliferación del establecimiento de compañías y pequeñas empresas en las localidades de Lorica y Cereté. Invirtiendo en los sectores del comercio, el agro, la minería y la ganadería, se encontraba Diego Martínez Camargo, quizás el empresario loriquero más importante para este periodo. Martínez Camargo creó fabricas de bebidas gaseosas, mantequilla, jabones, hielo, velas, leche, plantas eléctricas, de transporte fluvial, al tiempo que fundó compañías industriales para la explotación de recursos mineros y petroleros. De esta manera acumuló una significativa suma de capital que le permitió abrir sucursales en Cereté y establecer posteriormente su casa matriz en Cartagena, manteniendo una red de negocios que conectaba a esta ciudad con Lorica, Cereté en el Sinú y con Quibdó e Istmina en el Atrato. (Díaz 2004, p. 32 y Ripoll, 2008, pp.89-115).

\footnotetext{
${ }^{11}$ Biblioteca Bartolomé Calvo, (en adelante B.B.C), Libros de Contabilidad de la casa comercial Rafael del Castillo, 1870-1888-1890-1894-1895-1898-1900-1920.
} 
El otro espacio geográfico central en este circuito comercial era Quibdó que, para antes de la llegada de los inmigrantes sirio libaneses, ya mantenía una conexión con el puerto del Caribe a través de un pequeño grupo de comerciantes locales y cartageneros que realizaban negocios comerciales, agrícolas y mineros en la región. Como lo señala Luis Fernando González en su estudio sobre Quibdó $^{12}$, (González, 2003, p. 71). Los comerciantes del Chocó jugaron un papel decisivo en la época de la colonia para ciudades como Cali, Popayán y Cartagena, ciclo que se repite con los cartageneros a mediados del siglo XIX. En Quibdó, es notoria la actividad en el comercio de los hermanos Leoncio, Eladio y Federico Ferrer, quienes mantenían amplios nexos comerciales con Cartagena a través de la importación de mercancías, la exportación de recursos agrícolas y el negocio de maderas y muebles. Gonzalo Zúñiga, propietario de la firma comercial Zúñiga y Díaz, fue uno de los comerciantes de caucho y mercancías extranjeras entre Quibdó y Cartagena; así como Augusto y Luccindo Posso también mantenían un importante negocio de importación y exportación de mercancías, y se desempeñaban como prestamistas en Cartagena ${ }^{13}$.

La explotación y comercialización de recursos como el caucho, cacao, la tagua, las maderas y el platino, fueron las actividades de comerciantes locales como Lisímaco Andrade, Abraham Perea, Ricardo Valencia, Pedro Mosquera, Antonio Asprilla, Hortencio Ferrer, Camilo Mayo, entre otros ${ }^{14}$, pero sobre todo fueron éstas las que en mayor medida, junto a la navegación fluvial, desarrollaron los comerciantes cartageneros y sinuanos que mantenían vínculos con el Atrato. Bartolome Martínez Bosio y Diego Martínez, controlaron la navegación a vapor por el rio Atrato, mientras que firmas como Pombo Hermanos, Vélez Danies, Zubiria, Augusto Tono, Ismael Porto, Federico De la Espriella, se dedicaron a la exportación de platino, caucho y madera ${ }^{15}$, lo que convirtió a Cartagena en uno de los principales puertos exportadores de recursos forestales.

Es claro, entonces, que tanto en Cartagena y Quibdó como en Lorica y Cereté se hallaban una serie de comerciantes realizando actividades económicas, creando unas dinámicas internas que, al tiempo que fueron consolidando uno de los circuitos comerciales más importantes del Colombia, le imprimieron gran vitalidad a la vida comercial de las mencionadas poblaciones desde la segunda mitad del siglo XIX y primeros años del XX. Existía un circuito comercial que no solo logró mantener la vida económica de Cartagena, dinamizar la actividad comercial en las provincias del Atrato y el Sinú, reactivar un triangulo movilizador de una gran variedad de recursos agrícolas, forestales y mineros, sino que se

\footnotetext{
${ }^{12}$ En el año de 1854, un grupo de 17 vecinos de Quibdó emprendieron la iniciativa de la navegación fluvial.

${ }^{13}$ A.H.C. Fondo Gobernación, Protocolos Notariales, notaria primera, Cartagena, Escrituras $N^{\circ} 415,419,457$, 471, Cartagena, julio 2, 4, 15, 19 de 1919.

${ }^{14}$ Ibíd. p.p. 82, 142 y 143. B.N.C, “Avisos comerciales”, Los avisos, Quibdó, 14 agosto 1880, № 1 y 18 septiembre 1890, N 3. B.N.C, El Chocó, Quibdó, 1 febrero 1908. ABC. Quibdó, julio 7 de 1926.

${ }^{15}$ B.N.C, "Sueltos", Los visos, Quibdó, 10 junio 1891, №12 y 18 Junio 1891, №3. Ecos del Chocó, Quibdó, 5 agosto 1890, N2. Gaceta Departamental, Quibdó, 26 diciembre 1908, N 3 p. 3.
} 
construyó una importante red de negocios que configuraron una atractiva zona de interés comercial.

En este panorama, marcado por la existencia de un circuito comercial con unas actividades económicas ampliamente desarrolladas por un conjunto de empresarios locales y nacionales, es que se da la llegada de la ola inmigratoria a Colombia, cuya mayor expresión se registró entre las dos últimas décadas del siglo XIX y las tres primeras del siglo XX. De esta manera los inmigrantes arribaron a la región en medio de un contexto de auge económico, y así se les facilitó insertarse en las actividades comerciales de estas localidades, sacando provecho de ello al convertirse en parte activa del proceso de desarrollo económico de la región Caribe y el Atrato.

\section{El circuito se llena de sirio-libaneses}

La mayoría de los inmigrantes sirio-libaneses se habían establecido inicialmente en el puerto de Cartagena ${ }^{16}$, ciudad que para el año de 1912 contaba con una población de 36.632 habitantes y albergaba cerca de 865 extranjeros ${ }^{17}$, entre los que había un poco más de 500 sirio-libaneses. En su gran mayoría ubicados en el barrio de Getsemaní, donde tradicionalmente habitaba un significativo número de negros y mulatos excluidos de la sociedad cartagenera, inmigrantes sirio libaneses tales como los Rumie, Meluk, Abuchar, Chagui, Ganem, Malluk, entre otros, comenzaron a establecer casas y locales comerciales (Mendoza y Mendoza, 2007, pp.40-47), con las que no solo se establecieron en la sociedad cartagenera de la época, sino que también adelantaron un proceso de expansión comercial hacia las provincias del Sinú y el Atrato.

Efectivamente, Cartagena, al igual que Barranquilla, sirvió después como eje de apertura comercial y colonización hacia las diferentes poblaciones del interior de la región. (Cunninghame, 1920, p.231). De esta manera se fueron instalando en poblaciones como Lorica, Ceretè, Sahagún, Ciénaga de Oro, San Bernardo del Viento, Montería, Sincelejo, Tolú, Magangué, entre otros. En la Provincia del

\footnotetext{
${ }^{16}$ Los primeros nombres de este origen que se logran referenciar en la vida comercial y social del centro portuario de Cartagena y los puertos fluviales del Atrato y Sinú son Carlos, Miguel, Alejandro, Addo, Cesar, Salomón y Rafael Abuchar, del Libano y los hermanos Basilio, Assad, Camilo, Antonio y Luís Malluk, Miguel, Alejrandro, José, Abraham y Carlos Rumie, Amín, Tufik, Felix, Emilio, Bichyr y Kalil Meluk, Moisés Mebarak, Alejandro y José Dager, de Siria, Salim y Moisés Bechara, Salomón Ganem, Name H Morad, Moisés, Addo, Miguel, Antonio, José, Juan y Abraham Jattin, Checry Fayad, del Libano, Antonio Dumett, Elías Chaljud, Nicolás Char, Abraham Saker, Elías Saer, José Bechara, José Chaar, Salomón y José Chagui de Damasco- Siria, José Saibis, José y Miguel Calume, Elías y Milad Barguil, Fuad Cajale, Antonio y Jorge Bechara, Alfredo Chamat, Emilo Yurgaqui y Zaher, entre muchos más.

${ }^{17}$ Censo general de la Republica de Colombia. Levantado el 5 de marzo de 1912, presentado al congreso por el ministro de gobierno Pedro M Carreño, Bogotá, Imprenta Nacional, 1912.
} 
Atrato los inmigrantes sirio-libaneses se radicaron en poblaciones como Istmina, Condoto, Tadó, Acandí, Baudó, Quibdó.

Ubicándose en las diferentes poblaciones de estas provincias y dedicándose en su mayoría a la actividad comercial, este grupo de extranjeros fue uno de los más representativos dentro de los que hicieron presencia en estas provincias. De este origen parece que el primero en ubicarse en la zona del Sinú fue el libanés Moisés Jattin en 1880. (Behaine, 1980, p.146). Un grupo conformado por los Jattin, Chagui, Chaar, Abdala, Manzur, Behaine, Karduz, Gossain, Calume, entre otros, llegaron a Cartagena y posteriormente se ubicaron en poblaciones como Lorica y Ceretè, y otros marcharon hacia san Bernardo del Viento, Ciénaga de Oro y Montería ${ }^{18}$ (Ver mapa No.2).

Lorica, que para la época era la localidad más importante de la provincia por su privilegiada posición geográfica que la mantenía a un punto equidistante del mar y bañada por el río Sinú, principal vía de comunicación entre Cartagena y el interior de la provincia, mantuvo la bandera en cuanto a comercio e inmigración se refería. Su ubicación produjo tanto el movimiento frecuente de mercancías y productos agrícolas como el establecimiento de inmigrantes nacionales y extranjeros llegados a la provincia. Una lista de forasteros publicada en el año de 1929 en el periódico Renovación muestra la cantidad de personas foráneas que se encontraban establecidas en Lorica desarrollando actividades comerciales. En total agrupaban a 104 comerciantes que en su mayoría eran de Cartagena, Antioquia, otras poblaciones del Sinú y algunos extranjeros, siendo el grupo más representativo los comerciantes de origen sirio-libanés que unidos sumaban 40; es decir, los inmigrantes de esta nacionalidad representaban el 38.46 por ciento de los forasteros establecidos en Lorica ${ }^{19}$.

\footnotetext{
18 Entrevista realizada al señor Alberto Calume, descendiente directo, Cereté, 28 de febrero de 2004.

${ }^{19}$ A.H.C. "Lista de los forasteros de Lorica- comerciantes sirios", Renovación, Lorica, 5 de octubre de 1929.
} 
Mapa No. 2

Red de negocios de los sirio-libaneses en el circuito comercial (Cartagena - Sinú - Atrato)



Aunque Lorica era para entonces el centro obligado en el Sinú de la inmigración sirio-libanesa, Cereté también recibió un considerable grupo de estos inmigrantes, quienes desde el año de 1900 iniciaron su llegada a la población, instalándose en el comercio con la venta de telas, cacharrería y compra y venta de cueros y frutos. La población mantenía una actividad económica con Lorica, ya que la producción agrícola de su zona rural y sus alrededores se comercializaba 
en el casco urbano para su posterior salida hacia Lorica y Cartagena. Esta situación atrajo a inmigrantes sirios como los Char, Chagui, Calume, Barguil, Saibis, entre otros, quienes se establecieron en la población y para las primeras décadas del siglo XX estaban en la localidad representados en dieciséis (16) sociedades comerciales ${ }^{20}$.

En el Atrato, Quibdó era un centro administrativo, un punto de encuentro de caminos y arterias fluviales, que lo convirtieron en un importante centro de aprovisionamiento y comercio. Al estar bañado por uno de los ríos más navegables del Chocó, lo mantenía en una activa vida comercial con Cartagena, por lo que la presencia de sirios y libaneses también fue una realidad en esta población. Desde 1882 se puede referenciar la presencia de estos inmigrantes en las diferentes poblaciones de las provincias del Chocó (San Juan y Atrato). En Quibdó los primeros en llegar fueron los Abuchar, seguidos de los Meluk, Malluk, Rumie, Halaby, Bechara, Cajale, Chamat, Yurgaqui, Zaher y Ganem. Ya presentes en la realidad colombiana, los inmigrantes sirio-libaneses utilizarían diversas dinámicas para articularse a la realidad socio-económica de los distintos espacios donde se ubicaron, siendo el circuito comercial conformado entre la provincia del Sinú, Cartagena y la Provincia del Atrato, el escenario donde se hizo más notoria su presencia.

Los sirio-libaneses que se establecieron en el circuito comercial de Cartagena, la Provincia del Sinú y el Atrato, para el año de 1899 aparecían en avisos publicitarios en donde anunciaban las tiendas recién abiertas, las casas comerciales y las mercancías en oferta. Ya para este mismo año los nombres árabes aparecen con repetida frecuencia en los protocolos de algunas notarias, como en la notaria primera de Cartagena, donde se registró la formación de sociedades mercantiles, el otorgamiento de poderes y la constitución de hipotecas por miembros de las familias Fayad, Saab, Abuchar, Fadul, Aboshar y Meluk ${ }^{21}$.

Todas estas familias estaban vinculadas a la vida económica y comercial, como vendedores de mercancías, cultivadores, transportadores, importadores y exportadores de recursos agrícolas y mineros. Los sirio-libaneses se adentraron a este importante circuito, hasta el punto que ya para la década de los veinte formaban posiblemente el elemento más numeroso en la vida comercial y de negocios de los principales centros del circuito comercial (Posada \& Fawcett. 1998 , p. 38), con grandes logros en estos campos. Estos consiguieron fortalecer las actividades económicas, establecidas por los empresarios nacionales y locales, con su movimiento activo en los negocios, hasta el punto que supieron establecerse y en la mayoría de los casos monopolizar las actividades de comerciantes, exportadores, importadores y transportadores reconocidos. Diferentes escritores, diarios, informes y obras de la Costa a principios del siglo $\mathrm{XX}$, los destacan como parte constitutiva del comercio regional y como agentes, junto a los nacionales, de proyectos que contribuyeron al proceso de desarrollo

\footnotetext{
${ }^{20}$ Notaria única de Cereté, protocolos notariales, 1915- 1930.

${ }^{21}$ A.H.C., Notaria Primera de Cartagena, protocolos notariales, 1899-1900, cajas 100 a 125.
} 
económico y comercial de la región. P.L Bell, en su libro Colombia, a comercial and industrial handbook, destacaba en el año de 1921 que los inmigrantes siriolibaneses de manera progresiva estaban avanzando en el control de los negocios en la ciudad de Cartagena:

"Como un poderoso factor de mercado se encuentran los comerciante sirios en Cartagena, quienes compiten directamente con los viejos establecimientos colombianos. Aprovechando su sistema de agencias, sucursales y comercio con el interior, han sido capaces de ganar una buena mitad de los negocios del territorio". (Bell, P.L, 1921, p.212)

Una situación similar se presentaba en la región del Atrato, donde los siriolibaneses al iniciarse el siglo XX ya están referenciados como la colonia de inmigrantes más reconocida de Quibdó ${ }^{22}$ (González, 1997, p.70), a tal punto que para la segunda década de este siglo ellos son un grupo representativo en la lista de comerciantes establecidos en esta ciudad. En efecto, en el año de 1922, el $A B C$, periódico de mayor circulación en Chocó, publicó un directorio comercial destacando las 50 principales firmas comerciales radicadas en Quibdó, de las cuales 15 eran de propiedad de inmigrantes de origen sirio y libanes ${ }^{23}$. La escritora Soad Louis Laka, en su investigación sobre la inmigración árabe en el Caribe colombiano, logró referenciar 536 apellidos de este origen que se establecieron en Colombia, de este numero 382 se ubicaron en Cartagena, el Sinú y el Atrato (Louis, 2008, pp.37-50). Y una revisión a la prensa comercial y los protocolos notariales de la época permiten ver en mayor dimensión la cantidad de casas comerciales y empresarios de este origen que estaban establecidos como reconocidos comerciantes en la región del Caribe y el Atrato.

En el siglo XX los sirio-libaneses, a través de su participación en las actividades comerciales y la inversión en los sectores del agro, la ganadería, la minería y el transporte fluvial, no solo habían logrado integrarse exitosamente a la red comercial entre Cartagena y las provincias del Atrato y el Sinú, sino que su activo movimiento comercial se vio reflejado en el fortalecimiento de la actividad económica de este circuito comercial. El establecimiento de almacenes, casas comerciales y la constitución de empresas de transporte fluvial le imprimieron a este circuito, durante las primeras décadas del siglo XX, un aumento en las transacciones comerciales que se vio representado en la realización de compras y ventas de mercancías, productos agrícolas y mineros, tierras, ganado, la protocolización de préstamos e hipotecas y, sobre todo, en la constitución de empresas de navegación que mantenían un activo movimiento entre Cartagena y las provincias.

\footnotetext{
${ }^{22}$ A.H.C, Notaria Primera, Cartagena, protocolos notariales, 1880-1900.

${ }^{23}$ B.N.C, "Directorio comercial de Quibdó", $A B C$, Quibdó, 13 agosto 1922, № 706, p. 4. Ecos del Chocó, Quibdó, 30 noviembre 1907, p. 35.
} 


\section{Conclusión}

Uno de los circuitos comerciales más dinámicos a lo largo del siglo XIX y las primeras décadas del siglo XX en Colombia fue el conformado por Cartagena y las provincias del Sinú y el Atrato. Estos dos últimos espacios rurales jugaron un papel definitivo en el funcionamiento del mencionado circuito al convertirse en los principales proveedores de productos naturales, agrícolas, ganaderos y mineros que llegaban a Cartagena y luego redistribuidos hacia el interior del país y al exterior. Contrario a la caracterización que se ha hecho de la vida económica de las provincias que conformaban el circuito, como espacios carentes de dinamismo económico antes de la llegada de los sirio-libaneses, lo que parece estar presente es la activa vida comercial de espacios como Lorica, Cereté y Quibdó, donde un conjunto de empresarios nacionales y locales desarrollaron una amplia gama de negocios. Es precisamente en ese contexto de renglones económicos definidos y el funcionamiento del circuito comercial, donde los inmigrantes sirio-libaneses se establecieron y lograron insertarse en las dinámicas comerciales del circuito en estudio.

\section{FUENTES BIBLIOGRÁFICAS}

\section{Fuentes Primarias}

Prensa oficial y comercial de Quibdó -- B.N.C

Prensa oficial y comercial de Cartagena, Lorica y Cereté -- A.H.C

Fondo Gobernación, Notaria Primera de Cartagena, Protocolos 1880-1930 -A.H.C

Notarias únicas de Lorica y Cereté 1915-1930

Libros de Contabilidad de la casa comercial Rafael del Castillo, 1870-1888-18901894-1895-1898-1900-1920.-- B.B.C

\section{Libros y artículos}

Bejarano, Jesús Antonio. (1997). "El despegue cafetero (1900-1928)". En: Historia económica de Colombia. José Antonio Ocampo (compilador). Bogotá: ed. Biblioteca Familiar Presidencia de la República.

Behaine, Gladis. (1980). "Anotaciones sobre inmigraciones libanesas a Colombia". Bogotá: Revista Javeriana, №.467.

Bell, P.L. (1921). Colombia, a comercial and industrial handbook. Washington. 
Cuesta Moreno, Marco Tobias. (1986). El Chocó ayer, hoy y mañana, Bogotá: colección fundación publicaciones consigna.

Cunninghame, Graham. (1920). Cartagena y las riberas del Sinú, Montería: Ministerio de Agricultura, Incora.

Díaz, Fernando. (1994). Breve historia de Santa Cruz de Loríca. Bogotá: Tercer mundo editores.

. (2004) Cultura del Bajo Sinú: Tradición, educación y cambio. Santa Cruz de Lorica: Alcaldía Municipal de Lorica.

García, José Francisco. (1990, octubre- noviembre). "A raíz de los 250 años... ¿de dónde venimos?".Cereté: Notimes, 8.

González, Luis Fernando. (1997). "Sirio-libaneses en el Chocó, cien años de presencia económica y cultural”. En: Boletín Cultural y Bibliográfico, Vol. 34, № 44. Bogotá: Banco de la República.

(2003). Quibdó contexto histórico, desarrollo urbano y patrimonio arquitectónico. Bogotá: Universidad Nacional de Colombia.

Louis, Soad. (2008). Los inmigrantes árabes en los valles del Sinú, San Jorge, y otros destinos. Bogotá: Universidad de Córdoba, Plaza y Janes ed.

Machado, Adalberto. (1982). La exportación de carne y el parking house de coveñas, 1918-1930, tesis de grado. Cartagena: Universidad Tecnológica de Bolívar.

Mendoza, Ediverly \& Mendoza Obed. (2007). Actividades económicas de los inmigrantes sirio-libaneses en Cartagena 1920-1930, Tesis de grado para optar al título de historiador, Programa de Historia. Cartagena: Universidad de Cartagena.

Nacimento, Ayres. (1916-1919). Guía Ilustrada del Sinú. Montería: Tipografía El Esfuerzo.

Posada, Eduardo. (1998). El Caribe Colombiano una historia regional 1870-1950. Bogotá: Banco de la Republica/ ed. El Ancora.

Posada, Eduardo \& Fawcett Louise. (2000). "Árabes y judíos en el desarrollo del Caribe colombiano". En Boletín Cultural y Bibliográfico, Vol.35, № 49. Bogotá: Banco de la República. 
Restrepo, Jorge Alberto y Rodríguez, Manuel. (1986). "La actividad comercial y el grupo de comerciantes de Cartagena a finales del siglo XIX". En Estudios Sociales, № 1, Faes. Medellín.

Ripoll, María Teresa. (2000). "Redes familiares y el comercio en Cartagena: el caso de Rafael del Castillo \& Co, 1861-1960". En Cuadernos de Historia Económica y Empresarial, N. 5. Cartagena: Banco de la Republica.

(2008). Empresarios centenaristas en Cartagena cuatro casos de estudio. Cartagena: Banco de la República/ Universidad Tecnológica de Bolívar.

Solano, Sergio P. \& Conde, Jorge. (1993). Elite empresarial y desarrollo industrial en Barranquilla, 1875-1930. Barranquilla: Universidad del Atlántico.

Viloria, Joaquín. (2001) "Empresarios en Santa Marta: el caso de Joaquín y Manuel De Mier, 1800-1896”, en: Revista Aguaita, №.5.

(2003). "Lorica, una colonia árabe a orillas del Río Sinú". En Cuadernos de Historia Económica y Empresarial, No 10. Cartagena: Banco de la República (CEER).

Zambrano, Milton. (1998). El desarrollo del empresariado en la economía nacional 1880- 1945. Barranquilla: Fondo de publicaciones de la Universidad del Atlántico.

Recibido: Octubre 19 de 2011

Aprobado: Noviembre 29 de 2011 
